\title{
Potential of dynamic spectrum allocation in LTE macro networks
}

\author{
H. Hoffmann ${ }^{1}$, P. Ramachandra ${ }^{2}$, I. Z. Kovács ${ }^{3}$, L. Jorguseski ${ }^{4}$, F. Gunnarsson ${ }^{2}$, and T. Kürner ${ }^{1}$ \\ ${ }^{1}$ Deparmtent of Telecommunication Engineering, TU Braunschweig, Braunschweig, Germany \\ ${ }^{2}$ Ericsson Research, Linköping, Sweden \\ ${ }^{3}$ Nokia, Aalborg, Denmark \\ ${ }^{4}$ TNO, Delft, the Netherlands
}

Correspondence to: H. Hoffmann (hoffmann@ifn.ing.tu-bs.de)

Received: 12 December 2014 - Revised: 13 April 2015 - Accepted: 4 May 2015 - Published: 3 November 2015

\begin{abstract}
In recent years Mobile Network Operators (MNOs) worldwide are extensively deploying LTE networks in different spectrum bands and utilising different bandwidth configurations. Initially, the deployment is coverage oriented with macro cells using the lower LTE spectrum bands. As the offered traffic (i.e. the requested traffic from the users) increases the LTE deployment evolves with macro cells expanded with additional capacity boosting LTE carriers in higher frequency bands complemented with micro or small cells in traffic hotspot areas. For MNOs it is crucial to use the LTE spectrum assets, as well as the installed network infrastructure, in the most cost efficient way. The dynamic spectrum allocation (DSA) aims at (de)activating the available LTE frequency carriers according to the temporal and spatial traffic variations in order to increase the overall LTE system performance in terms of total network capacity by reducing the interference. This paper evaluates the DSA potential of achieving the envisaged performance improvement and identifying in which system and traffic conditions the DSA should be deployed. A self-optimised network (SON) DSA algorithm is also proposed and evaluated. The evaluations have been carried out in a hexagonal and a realistic sitespecific urban macro layout assuming a central traffic hotspot area surrounded with an area of lower traffic with a total size of approximately $8 \times 8 \mathrm{~km}^{2}$. The results show that up to $47 \%$ and up to $40 \%$ possible DSA gains are achievable with regards to the carried system load (i.e. used resources) for homogenous traffic distribution with hexagonal layout and for realistic site-specific urban macro layout, respectively. The SON DSA algorithm evaluation in a realistic site-specific urban macro cell deployment scenario including realistic nonuniform spatial traffic distribution shows insignificant cell throughput (i.e. served traffic) performance gains. Neverthe-
\end{abstract}

less, in the SON DSA investigations, a gain of up to $25 \%$ has been observed when analysing the resource utilisation in the non-hotspot cells.

\section{Introduction}

\subsection{Motivation}

The fast growing demand of mobile broadband services force mobile network operators (MNOs) to evolve their LTE networks from coverage oriented deployments towards capacity oriented deployment utilising macro cells with multiple LTE frequency carriers (at different LTE bands) complemented with small cells (micro, pico or femto cells) deployment in traffic hotspot areas. The network dimensioning is usually performed based on the estimated load in typical busy hour conditions. Therefore, although the deployed carriers are well utilised during peak hours, e.g. day time in university campus or offices, they become underutilised for the rest of the time, e.g. night time. At the same time the traffic demand may intensify in other areas of the network, e.g. in residential areas in the evening, located outside the busy hour high traffic areas. By means of Dynamic Spectrum Allocation (DSA) the available spectrum resources (LTE carriers) will be allocated according to the spatial and temporal traffic requirements by (autonomously) assigning spectrum to base stations based on the estimated large-scale temporal and spatial offered load (i.e. estimated amount of resources).

Furthermore, in LTE networks the DSA can be complemented by Interference Management (IM) mechanisms, such as the further/evolved Inter-Cell Interference Coordination (feICIC). Typical outcome of these interference management 
mechanisms is the (semi-)dynamic assignment of the available resources (time, frequency and/or power) between LTE macro and small-cell layers operating on the same carrier frequency.

The objective of our study is to identify the conditions under which a DSA mechanism could be utilised in an LTE macro network deployment in a typical European dense urban area.

\subsection{DSA in the academia}

A good overview of DSA approaches can be found in Akyildiz et al. (2006). The studies in Leaves et al. (2001, 2002, 2004) focus on spatial and temporal DSA in a multiradio network consisting of a UMTS and Digital Video Broadcasting-Terrestrial (DVB-T) system. A pre-requisite for the application of temporal DSA is load prediction. Simulation results for perfect and imperfect load prediction are presented showing that DSA has $30 \%$ higher spectrum efficiency compared to fixed channel assignment. The study in Rodriguez et al. (2006) extends the DSA concept with spectrum bidding among different cells from the participating DVB-T and UMTS systems. Further, Kovács and Vidács (2006) and Kovács et al. (2007) propose a spatial and temporal DSA, where the temporal DSA is coordinated by so called Regional Spectrum Brokers (RSB). The RSB considers interference by geographical and radio technology coupling parameters and solves the spectrum assignment problem using integer linear programming approach. The simulation results in Kovács et al. (2007) show that the combined RSB and linear programming optimisation approach achieve gains (e.g. $26 \%$ ) in terms of used resources over fixed spectrum assignment.

In Madan et al. (2011) a heuristic distributed algorithm is evaluated for dynamic sub-band partitioning and user associations complemented by transmit power control for LTE heterogeneous networks (macro overlaps with pico, femto and relay nodes). The heuristic algorithm uses light-weight coordination signalling messages between neighbouring (or overlapping) cells and provides significant throughput and delay gains over frequency re-use one.

\subsection{Standardisation and deployment status}

The related work on DSA within 3GPP can be divided into energy saving via switching on/off cells 3GPP TR36.887 (2014), 3GPP TR36.927 (2014) and inter-cell interference coordination (ICIC). The ICIC activities in 3GPP have started already in Release 8. In particular, for heterogeneous network scenarios the focus has been on the carrier based ICIC (CB-ICIC) for Release 10-11 that addressed scenarios for operational carrier selection, and downlink or uplink interference management for macro-pico as described in Qualcomm Inc. (2011) and 3GPP Overview (2013). Additionally, Release 12 also addresses the signalling needed for robust solutions for Release-10/11 UEs supporting carrier aggregation, and focus on solutions which do not require tight synchronisation between eNodeBs.

Today's MNOs operating GSM networks in $900 \mathrm{MHz}$ band (for coverage) and in $1800 \mathrm{MHz}$ band (for capacity) deploy DSA to dynamically switch ON and OFF the $1800 \mathrm{MHz}$ capacity cells according to the traffic demand for energy saving purposes, see e.g. Nokia Siemens Networks (2010). The control algorithm monitors the GSM traffic level and controls the activation of $1800 \mathrm{MHz}$ capacity layer by switching off the capacity cells when and where the traffic drops below certain pre-defined thresholds (usually at night hours). If the $1800 \mathrm{MHz}$ capacity layer is already deactivated the algorithm continues to monitor the traffic level and if needed activates back the $1800 \mathrm{MHz}$ capacity cells. Another practical example related to the DSA concept is trialled for wireless cellular communications in the public safety domain. The US public safety market is evolving rapidly with the combined introduction of public and private LTE networks. These networks have available spectrum that is often unused. Therefore, a dynamic spectrum arbitrage solution is trialled RadiSys Corporation (2013) that allows unused spectrum to be easily reallocated across networks to where it is needed most by combining prioritisation of users on the network with a real-time auctioning process.

\subsection{Scope of the study}

This study investigates the potential gain from DSA for a LTE macro-cellular network covering a geographical area with non-uniform spatial traffic distribution. The remaining of the paper is organised as follows. The system modelling approach and traffic assumptions are illustrated in Sect. 2. The potential DSA performance gains for a homogeneous traffic spatial distribution with hexagonal cell layout as well as with a site-specific urban cell deployment are presented in Sect. 3. Then, Sect. 4 presents the evaluation of the selfoptimised DSA algorithm in the site-specific urban deployment scenario. The paper is finalised with the conclusions and recommendations in Sect. 5.

\section{System modelling for DSA analysis}

For this study, we have selected an initial LTE carrier deployment within the allocated spectrum at a typical European LTE operator having $20 \mathrm{MHz}$ spectrum available in the $1800 \mathrm{MHz}$ band. For investigating the DSA potential two scenarios are considered, namely a hexagonal cell layout and a site-specific urban cell layout as illustrated in Fig. 1a and $\mathrm{b}$, respectively. Both scenarios use a traffic hotspot cell (one macro cell) in the centre of the investigated area illustrated with red colour in Fig. 1. The inter-site distance in the hexagonal layout was chosen such that the resulting cell density is 


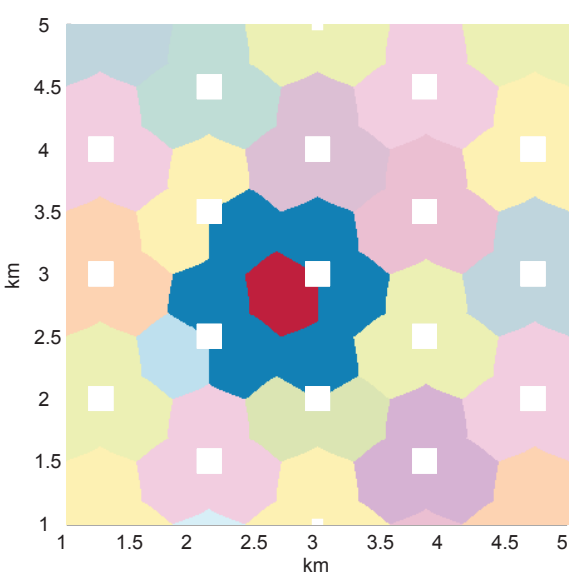

(a)

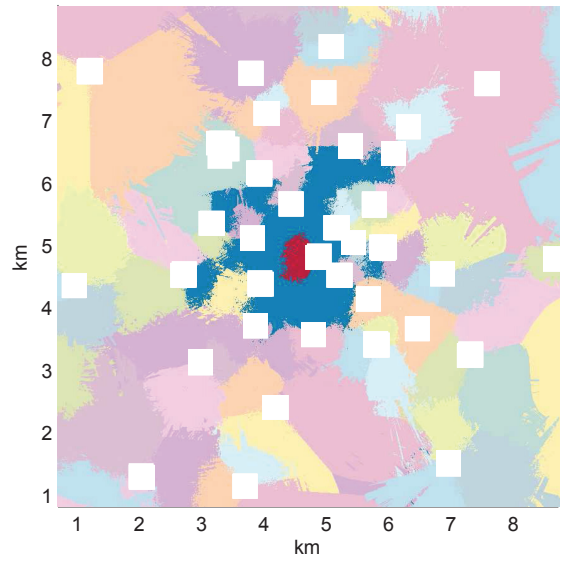

(b)

Figure 1. Cell layout (best server areas) with hotspot (red) and its surrounding cells (blue): (a) hexagonal layout scenario; (b) site-specific layout scenario (Hanover). The white square markers indicate the location of the macro sites.

Table 1. Macro network simulation parameters.

\begin{tabular}{lll}
\hline & $\begin{array}{l}\text { Hexagonal cell layout } \\
\text { (3GPP model) }\end{array}$ & $\begin{array}{l}\text { Site-specific cell layout } \\
\text { (Hanover urban network) }\end{array}$ \\
\hline Inter-site distance & $1000 \mathrm{~m}$ & $960 \mathrm{~m}$ (average) \\
Pixel size & $10 \mathrm{~m} \times 10 \mathrm{~m}$ & $10 \mathrm{~m} \times 10 \mathrm{~m}$ \\
Scenario area & $4 \mathrm{~km} \times 4 \mathrm{~km}$ & $8 \mathrm{~km} \times 8 \mathrm{~km}$ \\
Macro transmit power & $46 \mathrm{dBm}$ per cell & $46 \mathrm{dBm}$ per cell \\
Propagation model & 3GPP TS 25.184 & Ray-tracing based path loss \\
Traffic model & Uniform traffic intensity map & Spatially non-uniform distribution \\
& within each cell; separate & within and across cells; separate \\
Considered Cells for SINR computation & scaling for the hotspot cell & scaling for the hotspot cell \\
Cell selection & 20 strongest interfering cells & 20 strongest interfering cells \\
& Best signal server & Best signal server \\
\hline
\end{tabular}

similar to the one in the site-specific network layout in the considered area.

The requested traffic from the users in the network will be referred to as the offered traffic (to the network) in the following. The offered traffic levels in the hotspot cell and the surrounding cells are varied according to spatial traffic intensity maps that are scaled differently within the hotspot cell and the surrounding cells in order to generate different load ratios and interference situations to the area served by the hotspot cell. The traffic intensity maps are assumed to contain the average offered traffic in a certain time interval (e.g. $1 \mathrm{~h}$ ). Thus, there is no scheduling modelled explicitly and a time averaged resource allocation is simulated as explained in the following section. The main simulation parameters used in the evaluation of the DSA mechanisms are given in Table 1.

\subsection{DSA configurations}

In order to evaluate the potential DSA gains four different spectrum allocation strategies are investigated for the cells surrounding the hotspot cell, as given in Fig. 2. The bandwidth configuration, Full Spectrum is the situation without DSA mechanism activated i.e., all macro cells use the full bandwidth. Correspondingly, DSA-1, DSA-2, and Half Spectrum are configurations where only $17.2 \mathrm{MHz}(86 \%)$, $14.4 \mathrm{MHz}(72 \%)$, and $10 \mathrm{MHz}(50 \%)$ of the $20 \mathrm{MHz}$ spectrum is allowed to be used by the cells surrounding the hotspot cell, respectively. Note that the DSA-1 and DSA-2 restrict percentages of spectrum in use with a multiple of 1.4 $\mathrm{MHz}$ chunks (i.e. $2.8 \mathrm{MHz}$ restricted or $86 \%$ free for DSA-1 and $5.6 \mathrm{MHz}$ restricted or $72 \%$ free for DSA-2), which is the minimum LTE bandwidth. As a result a fraction of resources in the hotspot cell does not experience any interference from the surrounding cells. Implicitly, by employing this flexibility in bandwidth allocations a certain degree of interference management is achieved. 


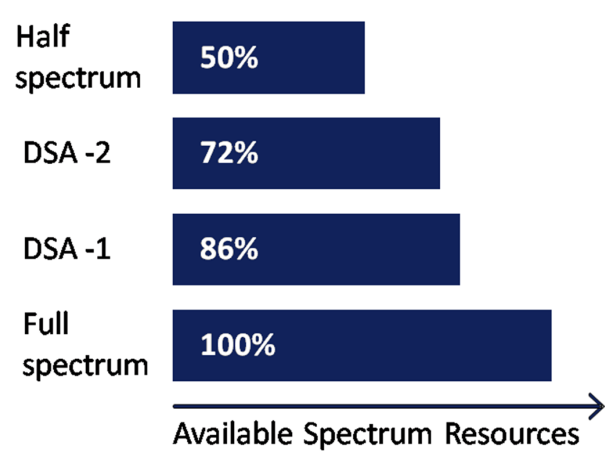

Figure 2. Resource level DSA method used in simulations.

\subsection{Traffic and resource allocation}

Serving cell selection is based on the best signal strength cell for each pixel of a predicted Reference Symbol Received Power (RSRP) map. A pixel is a square portion of the covered area characterized with its position and pixel area size, which is typically $10 \times 10 \mathrm{~m}^{2}$. The predicted RSRP map is a collection (or union) of all the pixels in the area under investigation where each pixel is associated with a predicted RSRP transmitted from a given LTE cell.

The amount of bandwidth $\left(\mathrm{BW}_{\mathrm{p} j}\right)$ required to serve the offered traffic (OfferedTraffic $\mathrm{p}_{j}$ ) in a pixel $\mathrm{p} j$ is dependent on the SINR experienced by the pixel $\left(\operatorname{SINR}_{\mathrm{p} j}\right)$ and is given by:

$$
\mathrm{BW}_{\mathrm{p} j}=\left\{\begin{array}{cc}
0 & \mathrm{SINR}_{\mathrm{p} 1}<\mathrm{SINR}_{\mathrm{min}}, \\
\frac{\text { OfferedTraffic }_{\mathrm{p} j}}{0.6 \cdot \log _{2}\left(1+\mathrm{SINR}_{\max }\right)} & \mathrm{SINR}_{\mathrm{p} 1} \geq \mathrm{SINR}_{\max }, \\
\frac{\text { OfferedTraffic }_{\mathrm{p} j}}{0.6 \cdot \log _{2}\left(1+\mathrm{SINR}_{\mathrm{p} j}\right)} & \text { otherwise, }
\end{array}\right.
$$

where $\mathrm{SINR}_{\min }=-6.5 \mathrm{~dB}$ and $\mathrm{SINR}_{\max }=22.05 \mathrm{~dB}$. These boundary SINR values model the cases where no transmission is possible or the highest modulation and coding scheme might be used, respectively. Note that the approach in this study uses average SINR values i.e. neglecting fast SINR variations over (multiple) $1 \mathrm{~ms} / \mathrm{TTI}$ periods. The total bandwidth required to serve all the pixels in the cell's coverage area is the sum of bandwidth requirements of each pixel (Eq. 1) in the coverage area and is given by

Total required bandwidth for a cell $l_{k}=\sum_{j \in \operatorname{cell}_{k}} \mathrm{BW}_{\mathrm{p} j}$.

Based on this value the CellLoad $k$ is modelled as:

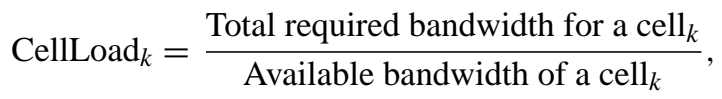

which is capped at $1(100 \%)$ for interference computation. It can also be seen as the used resources of a cell. If the to- tal required bandwidth in a cell is larger than the available bandwidth, then not all the offered traffic can be served, i.e.:

Throughput $_{k}=$ OfferedTraffic $_{k} \cdot \min \left(1, \frac{1}{\text { CellLoad }_{k}}\right)$.

\section{Evaluation of the potential DSA gains}

The evaluation in this section aims at quantifying the potential DSA gain in terms of total system carried traffic that can be served in the considered cells for the different DSA configurations described in Sect. 2.2.

The offered traffic in the surrounding cells and in the hotspot cell is varied in discrete steps for each of the DSA configurations listed in Fig. 2. The different load situations in the surrounding cells and the hotspot cell cause different interference situations, and hence different system performance. The carried traffic levels in the hotspot cell and the surrounding cells (given in traffic per cell) for the different DSA configurations are illustrated in Fig. 3. The curves present upper limits for the offered traffic such that all of the surrounding cells and the hotspot cell are loaded lower than the maximum cell load threshold of $100 \%$. Consequently, the offered traffic equals the carried/served traffic in the system. The left most values of the curves represent the maximum traffic that can be served in average in each of the surrounding cells with no or low load (and consequently no or low interference) from the hotspot cell. Note that the traffic in the surrounding cells for the hexagonal cell layout (see Fig. 3a) reaches a maximum of 20,17,14 and $10 \mathrm{Mbps}$ per cell for the Full Spectrum, DSA-1, DSA-2, and Half Spectrum DSA configurations, respectively. Note that these maximum traffic values scale with the amount of spectrum available in the surrounding cells. Correspondingly, for the site-specific deployment scenario (see Fig. 3b) the traffic in the surrounding cells reaches a maximum at $14,12,10$, and $6 \mathrm{Mbps}$ per cell. The lower values when compared to the hexagonal cell layout are due to the larger and irregular coverage areas resulting in also different interference conditions. As the offered traffic in the hotspot cell increases, the hotspot cell will cause more interference to the surrounding cells. Consequently, the increase in interference from the hotspot cell will cause the surrounding cells to get overloaded earlier, and their maximum carried traffic will gradually start to decrease. When the hotspot cell traffic crosses a threshold, the hotspot cell itself becomes overloaded and needs help from the surrounding cells to ensure that all the offered traffic is served. This help from the surrounding cells is in terms of reduction in their utilized spectrum so that they interfere less to the hotspot cell.

In Fig. 4 the gain in terms of total offered/carried system traffic values are illustrated for the different DSA configurations when compared with the Full Spectrum configuration. We can conclude that: 


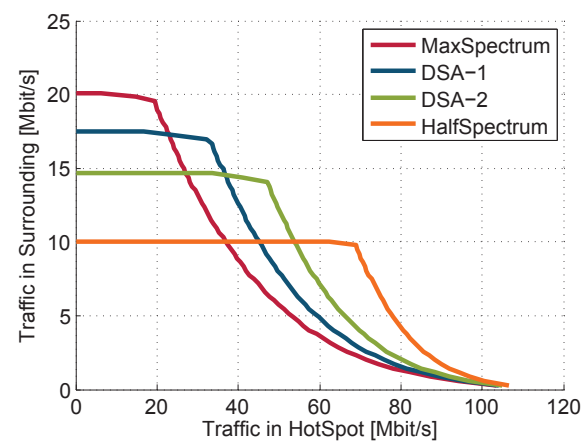

(a)

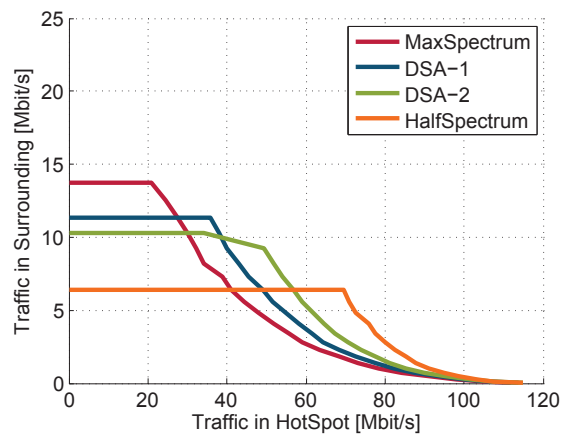

(b)

Figure 3. Served traffic in hotspot and the surrounding cells without cell overloading for different DSA configurations: (a) hexagonal layout scenario; (b) site-specific layout scenario (Hanover).

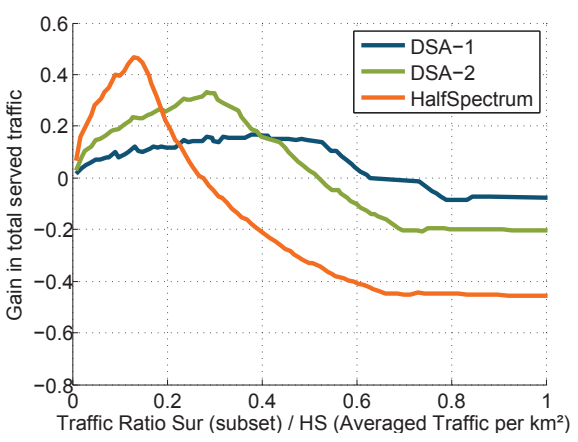

(a)

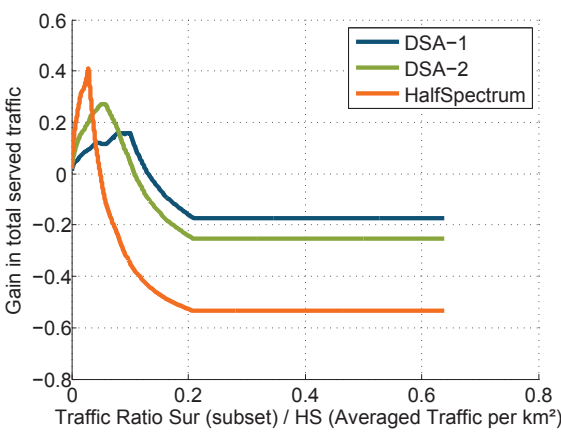

(b)

Figure 4. Gain relative to Full Spectrum in total carried system traffic for different ratios of surrounding (SUR) vs. hotspot (HS) traffic: (a) hexagonal layout scenario; (b) site-specific layout scenario (Hanover).

1. For the hexagonal layout (see Fig. 4a) up to $47 \%$ gain in terms of total served traffic can be achieved and the gain is visible in the traffic ratio range up to 0.6 . Furthermore, the optimal DSA configuration depends on the traffic ratio between the surrounding cells and the hotspot cell. For traffic ratios up to 0.2 the Half Spectrum DSA configuration is best while for the range of traffic ratios between 0.2 and 0.4 the $D S A-2$ is outperforming the other DSA configurations. For ratios higher than 0.4 DSA-1 configuration has the highest gain. An algorithm to allocate spectrum to cells dynamically would therefore take this ratio into account, as illustrated in Sect. 4.

2. For the site-specific urban macro deployment (see Fig. 4b) up to $40 \%$ gain in terms of total served traffic can be achieved and the gain is visible in the traffic ratio range up to 0.16 , which is significantly narrower range when compared to the hexagonal cell layout. Again, this is because of the larger and irregular cell area coverage creating different interference conditions.

\section{DSA evaluation for realistic layout and self-organised DSA algorithm}

Opposite to the uniform spatial traffic distributions used in Sect. 3, in the investigation of the realistic and self-organised DSA algorithm the offered traffic is based on scaled realistic traffic intensity maps for a selected time interval between 8:00 and 18:00 h, as explained in Deliverable 4.1 from SEMAFOUR D4.2 (2013). As the traffic intensity maps do not contain defined hotspots, a time-varying hotspot (per hour) is added to each of the hourly maps. For example, in the time interval between 11:00 and 15:00 $\mathrm{h}$ the traffic offered to the hotspot cell is approximately two times higher than the averaged offered traffic to its surrounding cells. The hotspot is placed in the coverage area of only one macro cell, as illustrated in Fig. 1b.

A flowchart of the SON enabled DSA algorithm that is executed in each cell is illustrated in Fig. 5. The DSA algorithm might change the spectrum only stepwise in pre-defined frequency chunks as described in Sect. 2.1, i.e. $20 \mathrm{MHz}$ (Full Spectrum), 17.2 MHz (DSA-1), $14.4 \mathrm{MHz}$ (DSA-2) and $10 \mathrm{MHz}$ (Half Spectrum). The DSA algorithm can be acti- 


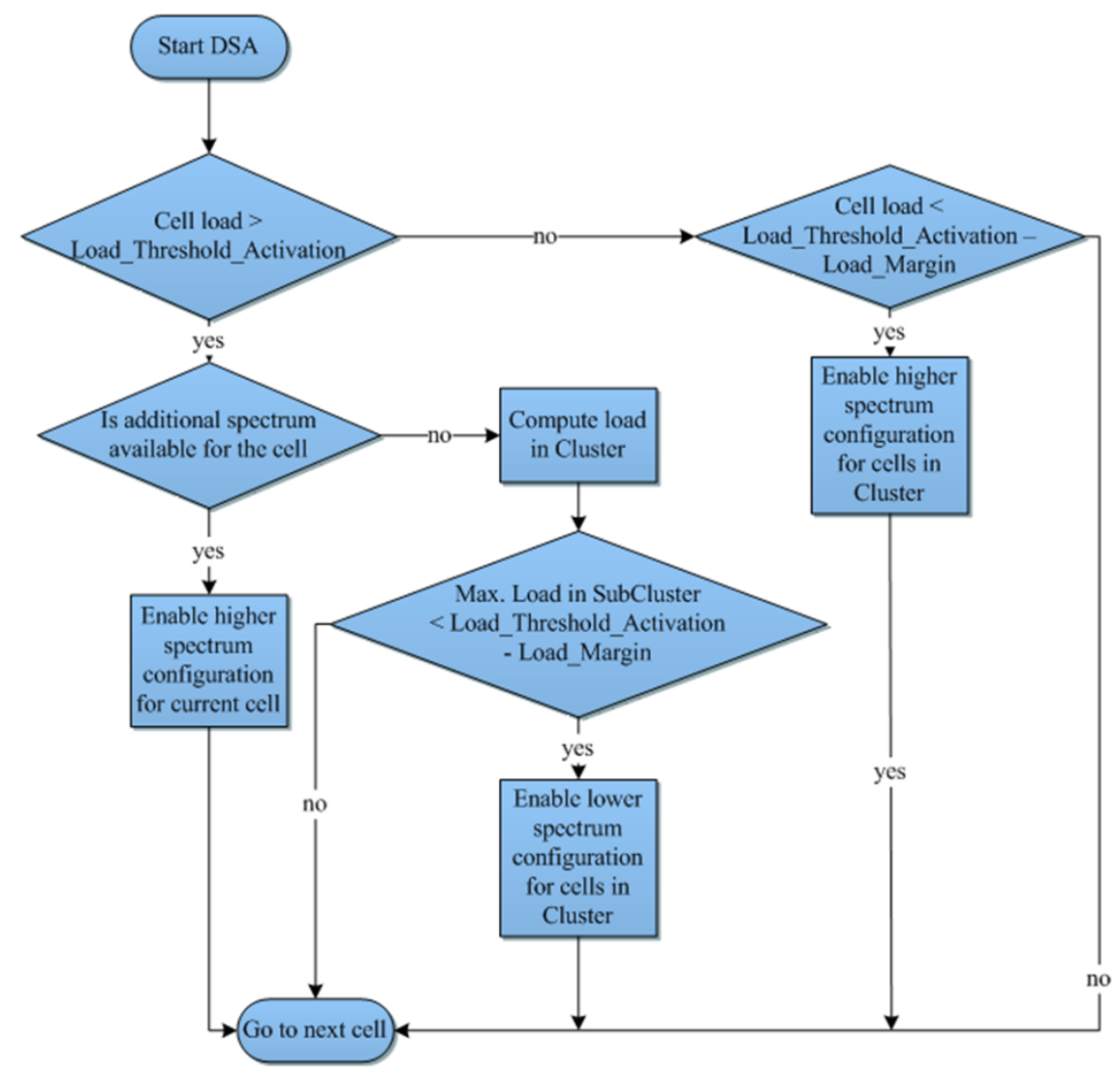

Figure 5. Self-optimised DSA algorithm flow chart.

vated in any cell and is triggered by a high load condition, as explained below. It is assumed that each cell has a preconfigured Cluster of interfering cells. In this study the top 20 interfering cells (surrounding the hotspot cell) are considered for the cluster.

The different load thresholds used in the flow chart in Fig. 5 can be explained from a cell and from the corresponding cluster neighbour perspective, as follows:

1. Load_Threshold_Activation: It is the load level in the cell above which the cell either requests for higher bandwidth configurations or requests its cluster neighbours to reduce their spectrum (not guaranteed of reduction yet as the actual reduction depends on the load levels in the neighbours).

\section{Load_Threshold_Activation-Load_Margin:}

a. From a cell perspective: When the load in the cell goes below this threshold, the cell allows its cluster neighbours to enable higher bandwidth configurations.

b. From the cluster cells' perspective: When the maximum load among the cell in the defined cluster neighbours goes below this threshold, they will ac- cept any request for further reduction in their bandwidth configurations.

The SON-enabled DSA algorithm evaluates every $20 \mathrm{~min}$ (i.e. three times per hour) the loads per cell and takes actions as defined in the flow chart in Figure 5. The values for the Load_Threshold_Activation and Load_Margin are set to 0.7 and 0.15 , respectively. In practice these parameters would be part of the overall DSA configuration in the network, and would be set by the MNO depending on the expected traffic variations, available spectrum, etc.

The performance results for the SON-enabled DSA algorithm are presented in Fig. 6. In the investigation the centre hotspot cell is always using $20 \mathrm{MHz}$ (full) spectrum. As it can be observed the DSA algorithm is triggered between 11:00 and 15:00 h, when the load in the hotspot cell increases above 0.7. The DSA algorithm then reduces the spectrum of the surrounding (top 20) cluster cells as long as the maximum load among the cells is below Load_Threshold_Activation $=0.7$.

It can be seen in Fig. 6 that cluster throughput curves for the simulation with DSA enabled and with DSA disabled are the same. The absence of DSA gain in terms of cluster throughput, when compared to the hexagonal layout and uniform traffic intensity per pixel, can be explained as follows: 


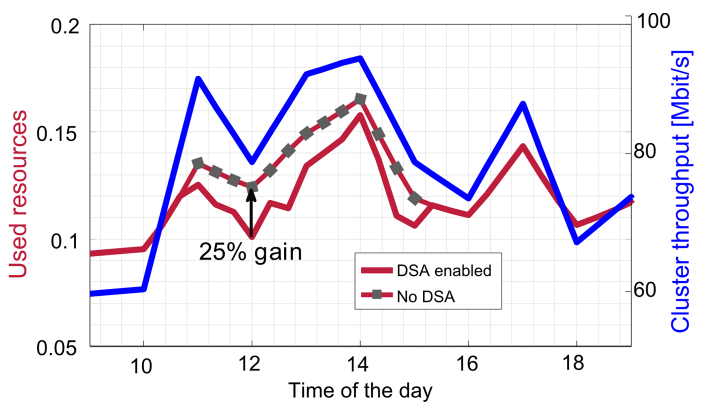

Figure 6. Performance of the self-optimised DSA algorithm in the site-specific layout scenario.

1. The non-uniform traffic intensity per pixel based on the realistic traffic intensity maps does not allow for very asymmetric traffic ratios between the hotspot cell and its corresponding cluster. Note that the high potential DSA gains for the site-specific deployment scenario (see Fig. 4b) were observed only in the traffic ratio range below 0.16

2. The SON enabled DSA algorithm cannot reduce the spectrum of the surrounding cluster cells down to halfspectrum configuration i.e. only DSA-1 and DSA-2 configurations are used, due to the relatively high traffic demand in the surrounding (top 20) cluster cells.

3. The non-uniform cell coverage areas i.e., largely different cell sizes when compared to the hexagonal layout, result in significantly different interference and cell load conditions when compared to the regular hexagonal layout.

In addition to the cluster throughput the corresponding (spectrum) resource utilisation is also plotted in Fig. 6. It can clearly be seen that the activation of the SON DSA algorithm achieves approximately the same cluster throughput while using significantly less resources. At the maximum (at 12 o'clock) there are $25 \%$ more resources used without DSA. These resource savings directly relate to the optimised interference situation in the network. Due to the restricted spectrum use in some of the cells pixels assigned to the remaining cells experience a higher SINR and thus require fewer resources.

\section{Conclusions}

This study investigated the potential performance gain from using dynamic spectrum allocation (DSA) in an LTE macrocellular network. The DSA evaluation has been carried out in a hexagonal cell layout with uniform spatial traffic distribution, as well as in a realistic site-specific cell deployment with non-uniform spatial traffic distribution scenarios. The centre cell was assumed to be a high traffic cell (hotspot) and always to utilise the total available spectrum of $20 \mathrm{MHz}$ while the spectrum allocation in surrounding cells was varied. Four different spectrum allocation strategies were used: Full Spectrum (20 MHz), DSA-l (17.2 MHz), DSA-2 (14.4 MHz) and Half Spectrum DSA (10 MHz).

The results show that DSA can provide significant gains only in spatially highly non-uniform traffic distributions. Under highly asymmetric traffic ratio between the surrounding cells and the hotspot cell, 0.5 or 0.16 for the hexagonal or realistic scenario, respectively, DSA gains up to $47 \%$ can be achieved. However, the same network configuration also lead to losses of about $50 \%$ when insufficient spectrum is allocated to the surrounding cells.

In the site-specific cell deployment the self-optimised DSA algorithm did not provide significant gain in the overall cell cluster throughput. This is because in the realistic network deployments the required highly asymmetric traffic between the hotspot and surrounding cells, when the full potential of DSA could be exploited, are difficult to achieve.

However, even though there is hardly any gain in cluster or cell throughput for the realistic network deployments, there is a significant gain of up to $25 \%$ in terms of resource utilisation. The free resources can be used for two purposes depending on the operator's policies. They can either be used for energy saving purposes, e.g. carriers can be switched off if a sufficient number of resources is unused or these free resources can be used as input for Multi-RAT DSA algorithms, i.e. they can be re-assigned to other RATs for capacity optimisation.

As a follow-up of this study the DSA gains for inter-RAT scenarios will be investigated for the case of LTE macro cells deployed in the same area with GSM macro cells. It is expected that DSA will be beneficial in dynamically re-farming parts of the allocated GSM spectrum as allowed by the ongoing GSM traffic and acceptable level of interference from the LTE cells re-using the same spectrum.

Acknowledgements. The research leading to these results has been carried out within the FP7 SEMAFOUR project and has received funding from the European Union Seventh Framework Programme (FP7/2007-2013) under grant agreement no. 316384.

Edited by: J. Anders

Reviewed by: two anonymous referees 


\section{References}

Akyildiz, I. F., Lee, W. Y., Vuran, M. C., and Mohanty, S.: $\mathrm{NeXt}$ generation/dynamic spectrum access/cognitive radio wireless networks: a survey, Comput. Netw., 50, 2127-2159, 2006.

Kovács, L. and Vidács, A.: Spatio-temporal spectrum management model for dynamic spectrum access networks, in: Proceedings of the first international workshop on Technology and Policy for Accessing Spectrum (TAPAS 2006), ACM, New York, NY, USA, 5 August, 2006.

Kovács, L., Vidács, A., and Tapolcai, J.: Spatio-Temporal Dynamic Spectrum Allocation with Interference Handling, IEEE Conf. Comm., Glasgow, UK, 24-28 June 2007, 5575-5580, 2007.

Laselva, D.,Altman, Z., Balan, I., Bergström, A., Djapic, R., Hoffmann, H., Jorguseski, L., Kovács, I. Z., Michaelsen, P. H., Naudts, D., Ramachandra, P., Sartori, C., Sas, B., Spaey, K., Trichias, K., and Wang, Y.: SON functions for multi-layer LTE and multi-RAT networks (first results), INFSO-ICT-316384 SEMAFOUR, Report, 141 pp., 2013.

Leaves, P., Ghaheri-Niri, S., Tafazolli, R., Christodoulides, L., Sammut, T., Staht, W., and Huschke, J.: Dynamic spectrum allocation in a multi-radio environment: concept and algorithm, 2nd International Conference on 3G Mobile Communication Technologies, London, UK, 26-28 March 2001, Conf. Publ. No. 477, 53-57, 2001.

Leaves, P., Huschke, J., and Tafazolli, R.: A summary of dynamic spectrum allocation results from DRiVE, IST Mobile and Wireless Telecommunications Summit, Thessaloniki, Greece, 17-19 June 2002, 245-250, 2002.
Leaves, P., Moessner, K., Tafazolli, R., Grandblaise, D., Bourse, D., Tönjes, R., and Breveglieri, M.: Dynamic spectrum allocation in composite reconfigurable wireless networks, IEEE Commun. Mag., 42, 72-81, 2004.

Madan, R., Borran, J., Sampath, A., Bhushan, N., Khandekar, A., and Tingfang, J.: Cell Association and Interference Coordination in Heterogeneous LTE-A Cellular Networks, IEEE J. Sel. Area Comm., 28, 1479-1489, 2010.

Nokia Siemens Networks: Smart Energy Control cuts $\mathrm{CO}_{2}$ footprint and saves up to $1.2 \mathrm{GWh}$ of power annually, available at: http://nsn.com/portfolio/customer-successes/success-stories/ smart-energy-control-cuts-co2-footprint-and-saves-up-to (last access: 19 May 2015), 2010.

RadiSys Corporation: Radisys LTE Solutions Enable Rivada Networks' Technology to Dynamically Allocate Excess Spectrum for Public Safety Networks, available at: http://www.radisys.com/2013/ (last access: 19 May 2015), 2013.

Rodriguez, V., Moessner, K. K., and Tafazolli, R.: Market driven dynamic spectrum allocation over space and time among radioaccess networks: DVB-T and B3G CDMA with heterogeneous terminals, Mobile Netw. Appl., 11, 847-860, 2006.

Qualcomm Inc.: R3-112609, Carrier-based HetNet ICIC use cases and solutions, The 3rd Generation Partnership Project (3GPP), 2011.

The 3rd Generation Partnership (3GPP): Overview of 3GPP Release 12 V0.1.0, 3GPP, available at: http://www.3gpp.org/ specifications/releases/68-release-12 (last access: 19 May 2015), 2013. 